\title{
FEASIBILITY OF PALIPERIDONE FOR TRANSDERMAL THERAPEUTIC SYSTEMS: EX VIVO PERMEATION KINETIC STUDIES OF DRUG THROUGH RAT ABDOMINAL SKIN
}

\author{
SADASHIVAIAH R, ROHITH G, SATHEESHA BABU BK*
}

Department of Pharmaceutics, Government College of Pharmacy, Bengaluru, Karnataka, India. Email: bksatishbabu@gmail.com

Received: 09 July 2019, Revised and Accepted: 01 August 2019

ABSTRACT

Objective: The main objective of the current research study was to investigate the effect of various permeation enhancers on the skin permeation of paliperidone for transdermal therapeutic systems (TTS).

Methods: A part of pre-formulation studies was performed to authentication of the drug by determining the melting point, solubility, partition coefficient (PC), attenuated total reflection-Fourier transform infrared, differential scanning calorimetry, and its purity by reversed-phase high-performance liquid chromatography. Ex vivo permeation kinetic study for paliperidone alone and with 5\% concentration of permeation enhancers hyaluronidase, dimethylsulfoxide (DMSO), groundnut oil, and tween 80 was conducted in modified Franz diffusion cell through rat abdominal skin as a barrier. The receptor phase containing $20 \%$ polyethylene glycol 400 in normal saline was maintained at $37^{\circ} \mathrm{C}$. The steady-state flux was obtained to calculate permeability coefficient, enhancement ratio (ER), and the cumulative amount of drug permeated at $12 \mathrm{~h}$

Results: The pre-formulation study results indicated that the received pure drug was authentified as paliperidone and its purity at par with official pharmacopeia. The PC of the drug was found to be $1.916 \pm 1.07$, indicated that the drug to be lipophilic. The ex vivo permeation study results showed that the enhancement effect of some permeation enhancers on paliperidone was as follows: Hyaluronidase>DMSO>groundnut oil $>$ tween 80 . Hyaluronidase has high permeation enhancing activity with the highest permeation flux of $12.038 \mu \mathrm{g} / \mathrm{cm}^{2} / \mathrm{h}$, and the cumulative amount of drug permeated was $212.760 \mu \mathrm{g} / \mathrm{cm}^{2}$. The ER of hyaluronidase was 3.69 folds higher than control.

Conclusion: The results of the present research study attributed that hyaluronidase was a potential permeation enhancer which would be included in the TTS of paliperidone.

Keywords: Paliperidone, Antipsychotic drug, Permeation enhancers, Kinetics, Differential scanning calorimetry, Reversed-phase high-performance liquid chromatography, Hyaluronidase.

(C) 2019 The Authors. Published by Innovare Academic Sciences Pvt Ltd. This is an open access article under the CC BY license (http://creativecommons. org/licenses/by/4. 0/) DOI: http://dx.doi.org/10.22159/ajpcr.2019.v12i10.34836

\section{INTRODUCTION}

The total surface area of the skin is about $1.858 \mathrm{~m}^{2}$ and is the biggest body organ. The delivery of the drug through the skin portal to systemic circulation at a predetermined rate and maintain clinically the effective concentrations over a prolonged period [1]. Transdermal patches are a common route of administration for hormonal therapy, narcotic analgesia, neurological disorders, and hypertension [2], and they need prolonged administration of drugs to keep up drug concentration at a therapeutic level. Presently, more than 35 transdermal patches have been approved for sale in the United States, and globally more than 16 active pharmaceutical ingredients are approved for use $[3,4]$

Transdermal therapeutic systems (TTS) provide many potential advantages, non-invasive route of administration, avoidance of hepatic first-pass effect, elimination of gastrointestinal (GI) irritation, decreased toxicity, fewer side effects as well as greater patient compliance [5]. The major disadvantage of TTS is the barrier property of the skin and low permeability of some drugs. Hence, the greater challenge to develop TTS is to make improved permeability of drugs to the skin [6]. Several techniques have been considered to enhance the permeation of drugs [7]. Permeation enhancers are one of the most convenient methods and show relatively strong effects than other enhancement techniques [8].

Permeation enhancers are agents that enhance the skin permeability of low permeable compounds by altering the characteristics of skin or barrier to achieve desired flux. In our study, four different permeation enhancers were used, they are hyaluronidase, dimethylsulfoxide
(DMSO), groundnut oil and tween 80 , at 5\% concentrations on the permeation of paliperidone. Permeation enhancers are incorporated into a formulation to improve the diffusivity and solubility of drugs through the skin that would reversibly reduce the barrier resistance of the skin [9]. Enhance the permeation of drugs by the combination of mechanisms including disruption of the lipid bilayer, fluidization, and extraction of stratum corneum lipids [10] thus allows the drug to penetrate the viable tissues and enter the systemic circulation.

The atypical antipsychotic drug, paliperidone belongs to benzisoxazole derivative and is a metabolite product of risperidone [11]. Paliperidone is formulated as oral and parenteral dosage forms for the treatment of schizophrenia and schizoaffective disorder [12], which is incompletely absorbed from GI tract with oral bioavailability of $28 \%$. Besides, it has a low dose of 1.5-9 mg/d with high oral side effects [13]. Lead to the selection of drug to formulate as TTS. This current research work was to investigate the feasibility of paliperidone for TTS and to test the effect of various permeation enhancers that would be incorporated into a TTS consisting of paliperidone.

\section{MATERIALS AND METHODS}

\section{Materials}

Chemicals

Paliperidone working standard and pure drug (active pharmaceutical ingredient) were received as a gift sample from BAL Pharma Ltd., Bengaluru, Karnataka, India. Methanol, acetonitrile, and DMSO were purchased from Merck Specialists Pvt., Ltd., Mumbai. Hyaluronidase was purchased from Shreya Life Sciences Pvt. Ltd., Bengaluru. Tween 
80 (HiMedia), polyethylene glycol (PEG) 400, groundnut oil, and sodium chloride were procured from a local supplier, Bengaluru. High-performance liquid chromatography (HPLC) grade water and distilled water were prepared using Milli- $Q^{\circledR}$ Millipore water purification system.

Animals

Albino rats of either sex weighing 100-200 g (age 8-12 weeks) were used in ex vivo skin permeation study. Rats were received from the animal house, Department of Pharmacology, Government College of Pharmacy, Bengaluru. For this study, the approval was obtained from the IAEC (Institutional Animal Ethics Committee), Government College of Pharmacy, Bengaluru (IAEC Reference No.: DCD/GCP/20/E.C/ ADM/2017-2018). Before the experiment started, the animals were fed food and water ad libitum.

\section{Methods}

Authentication of paliperidone

Melting point

The melting point of paliperidone was determined using MR-VIS visual melting range apparatus (Labindia Analytical Instruments Pvt., Ltd., India) by the capillary method.

\section{Solubility study}

The solubility of paliperidone in different solvents were determined. In this method, the saturated solutions of paliperidone were prepared by adding an excess amount of drug into $10 \mathrm{ml}$ of solvents. The saturated solutions were sonicated in a temperature-controlled water bath for $6 \mathrm{~h}$ at $25 \pm 0.5^{\circ} \mathrm{C}$. After the specified period, the saturated solutions were filtered through a $0.45 \mu \mathrm{m}$ Whatman nylon membrane filter [14]. The concentration of the drug in the solvents was determined by making suitable dilutions and analyzed using reversed-phase-HPLC (RP-HPLC) ( $\mathrm{n}=3$ ).

\section{Partition coefficient (PC)}

The PC of the paliperidone was determined by taking an equal portion of n-octanol (organic phase) and water (aqueous phase) in a separating funnel by a shake-flask method. The pre-saturation was achieved by thorough mixing of both the phases for $10 \mathrm{~min}$ at room temperature and then let them stand long enough to allow the phases to achieve the saturation state. $10 \mathrm{mg}$ of the drug was added, and it was shaken for $45 \mathrm{~min}$ and allowed for $1 \mathrm{~h}$ to separate two distinct phases. Finally, aqueous and organic layers were separated. Centrifuged at $1000 \mathrm{rpm}$ for $5 \mathrm{~min}$, the concentration of the drugs in each separated phase was determined by making suitable dilutions and analyzed by RP-HPLC at $237 \mathrm{~nm}$ after passing through $0.45 \mu \mathrm{m}$ Whatman nylon membrane filters [15]. The PC $(\log o / w)$ of paliperidone was calculated by the following formula:

$$
\mathrm{PC}=\mathrm{Co} / \mathrm{Cw}
$$

Where Co is the concentration of the drug in n-octanol and Cw is the concentration of the drug in water.

\section{Attenuated total reflection-Fourier transform infrared (ATR-FTIR)} analysis

ATR-FTIR spectroscopy studies were performed in Shimadzu IR Affinity-1S (Shimadzu Corporation, Japan). The paliperidone drug was directly placed on the germanium prism and FTIR spectra were recorded in the scanning measurement range of $600-4000 / \mathrm{cm}$ [16].

\section{Differential scanning calorimetry (DSC) analysis}

1-3 mg of paliperidone sample was placed in aluminum pans and pans were closed by crimping the lids. Filled aluminum pans and empty sealed pan were placed in a sample holder where empty pan acts as a reference. The thermal behavior of the drug sample was investigated under nitrogen purging at a scanning rate of $10^{\circ} \mathrm{C} / \mathrm{min}$ [17], covering the temperature range of $30-350^{\circ} \mathrm{C}$ and the samples were analyzed using DSC-8000 (PerkinElmer Inc. Germany).

\section{Purity determination}

RP-HPLC analysis

Assay purity of received drug was confirmed by RP-HPLC. The analysis of paliperidone was determined by RP-HPLC using an in-house developed and validated method. A typical chromatographic condition for the determination of the content of paliperidone in the pure drug consisted of Shimadzu-LC-20AD Prominence (Shimadzu Corporation, Japan). The separation was achieved on a Gemini NX C18 column (Phenomenex-150 $\mathrm{mm} \times 4.6 \mathrm{~mm}, 5 \mu \mathrm{m}$ ) eluted with a mobile phase composing of methanol:acetonitrile:buffer solution $(\mathrm{pH} 6.0)$ in the ratio of 50:20:30 v/v. The flow rate of $1.0 \mathrm{ml} / \mathrm{min}$, UV detection wavelength $237 \mathrm{~nm}$ and injection volume $20 \mu \mathrm{l}$, filtered through $0.45 \mu \mathrm{m}$ finer porosity nylon membrane filter and degassed before use. Methanol was used as a diluent. The typical retention time was $3.2 \mathrm{~min}$ and the total run time was 7 min. Peak area was used to estimate the paliperidone concentration against the working standard peak area.

\section{Ex vivo permeation kinetics study}

Apparatus

Ex vivo permeation studies were carried out using modified Franz diffusion cells (slight modifications in the Franz diffusion cell), made up of borosilicate glass, with $3.462 \mathrm{~cm}^{2}$ diffusional surface area. The donor and receptor compartments were held together using spring. The content of receptor cells was stirred by placing the diffusion cells on 10 channel magnetic hotplate stirrer (DLab MS-H-S-10, LabCon, USA) using a star head-shaped magnetic bead (Serwell instruments, India) rotating at $100 \mathrm{rpm}$. The temperature of the content of the receptor compartment was maintained at $37 \pm 0.5^{\circ} \mathrm{C}$ by circulating hot water through the jacketed receptor compartment by a peristaltic pump.

\section{Preparation of rat skin}

The full thickness of the abdominal skin was obtained from albino rats. The rat was anesthetized using chloroform, and long hairs were cut with the help of scissors and shaved [17]. Using scalp vein the abdominal skin was removed, and the skin was freed from adhering subcutaneous fat by dipping in hot water at $55^{\circ} \mathrm{C}$ for $30 \mathrm{~s}$ than using fresh distilled water. The skin was washed and immersed in a receptor fluid for 5 min after the skin was thoroughly inspected visually for its integrity and stored at $-20^{\circ} \mathrm{C}$ until it is used [18]

\section{Experiment}

The ex vivo skin permeation kinetics of paliperidone were studied with the drug alone and with hyaluronidase, DMSO, groundnut oil, and tween 80 as permeation enhancing agents. The permeation study was conducted in a modified Franz diffusion cell. The barrier used was the prepared rat skin after bringing the temperature form DLab $-20^{\circ} \mathrm{C}$ at room temperature by placing the skin in a 20\% PEG 400 in normal saline which acts as a receptor medium for $1 \mathrm{~h}$. The receptor compartment was filled with a receptor medium. The prepared skin was placed between the receptor and the donor compartment considering the stratum corneum facing the donor compartment. The donor compartment containing a $5 \mathrm{ml}$ drug solution $(0.5 \mathrm{mg} / \mathrm{ml})$ was placed on the skin with the dermal side in contact with the receptor phase. To achieve the sink condition, the content of the receptor compartment was stirred at rpm of 100 , and fresh medium was replaced by the volume of aliquots removed from the receptor compartment. $1.5 \mathrm{ml}$ of aliquots were withdrawn at predetermined intervals of $1,2,3,4,5,6,7,8$, and $12 \mathrm{~h}$ from the sampling port [19]. Care must be taken during the withdrawal of aliquots no air bubble formation in the receptor compartment which would reduce the effective permeation area. After appropriate dilution, the samples were analyzed by RP-HPLC $(n=3)$.

\section{Data analysis}

Steady-state flux (Jss) was obtained by plotting the cumulative amount of drug alone and along with permeation enhancers permeated $\left(\mu \mathrm{g} / \mathrm{cm}^{2}\right)$ against time in $\mathrm{h}$ and slope was considered as a steady-state flux (mass/area/time). To get permeability coefficient (Kp), the steady-state flux was divided by the concentration of drug in donor 
compartment at time zero, which is an indication of the velocity of drug permeated through the barrier in $\mathrm{cm} / \mathrm{h}$. The permeation enhancing effect of permeation enhancer was calculated based on Kp in terms of enhancement ratio (ER). The Jss, Kp, and ER are calculated using the Equations 1, 2, and 3, respectively [20]:

$$
\mathrm{Jss}=\mathrm{Q} /(\mathrm{A}-\mathrm{t})
$$

Where, $Q$ is the quantity of drug traversing the skin membrane in time $t$, and $\mathrm{A}$ is the area of exposed skin in $\mathrm{cm}^{2}$.

$$
\mathrm{Kp}=\mathrm{Jss} / \mathrm{C}
$$

Where, $\mathrm{C}$ is the concentration of drug in the donor compartment at time zero.

$\mathrm{ER}=\mathrm{Kp}$ with permeation enhancer $/ \mathrm{Kp}$ without permeation enhancer (3)

\section{RESULTS AND DISCUSSION}

\section{Melting point}

The melting point of paliperidone was found to be $182.8^{\circ} \mathrm{C}$ which is compared with the available data in the standard literature [21] and also complied with official standard data that are in the range of $181^{\circ} \mathrm{C}-186^{\circ} \mathrm{C}$

\section{Solubility studies of paliperidone}

The solubility of paliperidone in water was found to be $0.032 \mathrm{mg} / \mathrm{ml}$, indicates poor solubility. The solubility of paliperidone observed in $0.1 \mathrm{~N} \mathrm{HCl}$, methanol, PEG 400, DMSO, and tween 80 was found to be $5.053 \pm 0.87,2.888 \pm 1.81,0.622 \pm 0.51,2.142 \pm 0.77$, and $0.052 \pm 0.67 \mathrm{mg} / \mathrm{ml}$, respectively. These values are compared with the available data in the standard literature. Among the solvents tested, methanol and PEG 400 could be a better choice of cosolvent in the medium $[22,23]$.

\section{PC}

The PC value of paliperidone in n-octanol and water system was found to be $1.916 \pm 1.07$. The reported value is within the limits of -1 to 4 as required for the transdermal permeation [24].

The obtained value suggested that the drug possesses enough lipophilic property, which meets the requirements to formulate it into a transdermal therapeutic system.

\section{ATR-FTIR analysis}

The ATR-FTIR spectra of paliperidone are shown in Fig. 1. The paliperidone exhibited sharp characteristic peaks at $864.11 \mathrm{~cm}^{-1}$ for C-H (aromatic) bend, $956.69 \mathrm{~cm}^{-1}$ for C-O stretch, $1132.21 \mathrm{~cm}^{-1}$ for C-F stretch, $1340.53 \mathrm{~cm}^{-1}$ for C-N stretching, $1406.11 \mathrm{~cm}^{-1}$ for $\mathrm{C}-\mathrm{H}$ bend, $1527.62 \mathrm{~cm}^{-1}$ for $\mathrm{C}=\mathrm{N}$ stretch, $1620.21 \mathrm{~cm}^{-1}$ for $\mathrm{C}=0$ stretch, and $3294.42 \mathrm{~cm}^{-1}$ for $\mathrm{C}-\mathrm{OH}$ stretching. All these characteristic peaks were also found in the working standard spectra and available data in standard literature [21].

\section{DSC analysis}

The DSC thermogram of paliperidone is shown in Fig. 2 a sharp endothermic peak at $185.52^{\circ} \mathrm{C}$. The DSC thermogram of paliperidone peak value determined was concurrent with standard literature value [21,25].

Based on the above results obtained from the ATR-FTIR and thermal analysis (melting point and DCS) techniques, it can be indicated that the received drug was authentified as paliperidone.

\section{Determination of drug purity by HPLC analysis}

The purity of paliperidone was determined by an in-house developed and validated RP-HPLC method. The purity of paliperidone was found to be $99.84 \%$ on the dried basis, its purity at par with working standard assay purity value and USP (acceptance criterion: NLT 98.0\% and NMT $102.0 \%$ of paliperidone, calculated on the dried basis). A typical HPLC chromatograph of paliperidone is shown in Fig. 3.

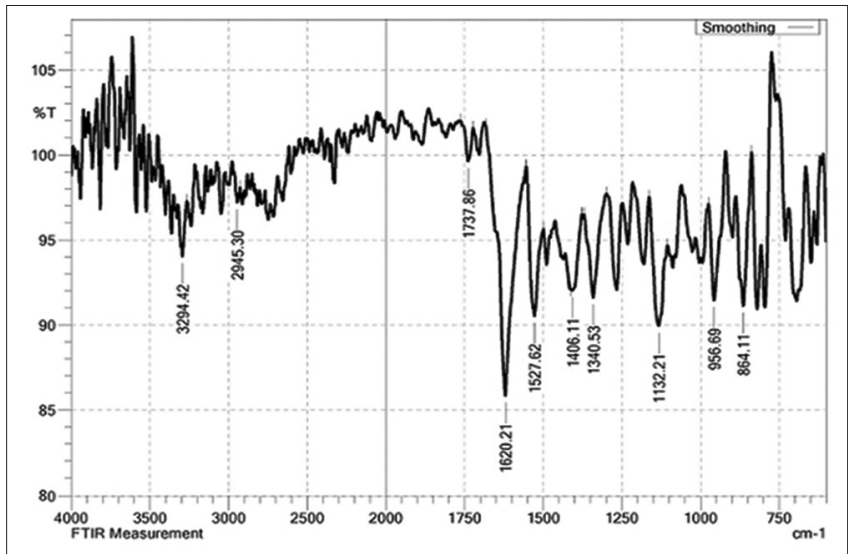

Fig. 1: Attenuated total reflection-Fourier transform infrared spectra of paliperidone

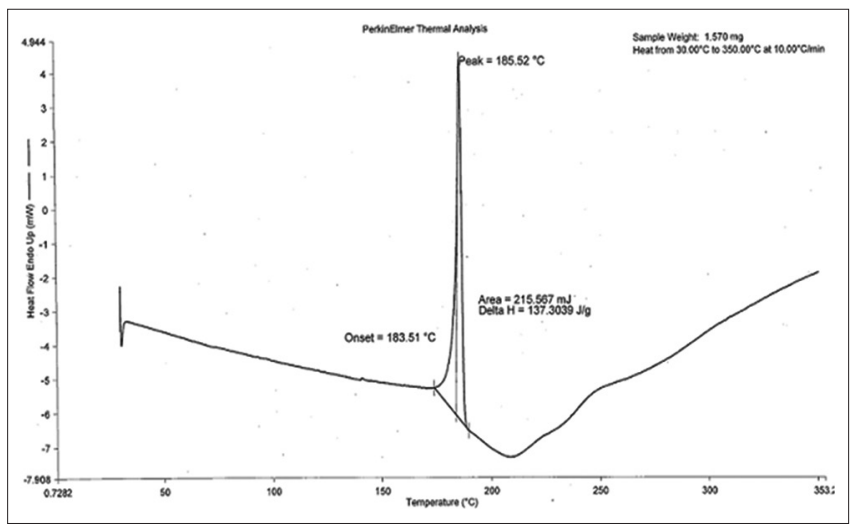

Fig. 2: Differential scanning calorimetry thermogram of paliperidone

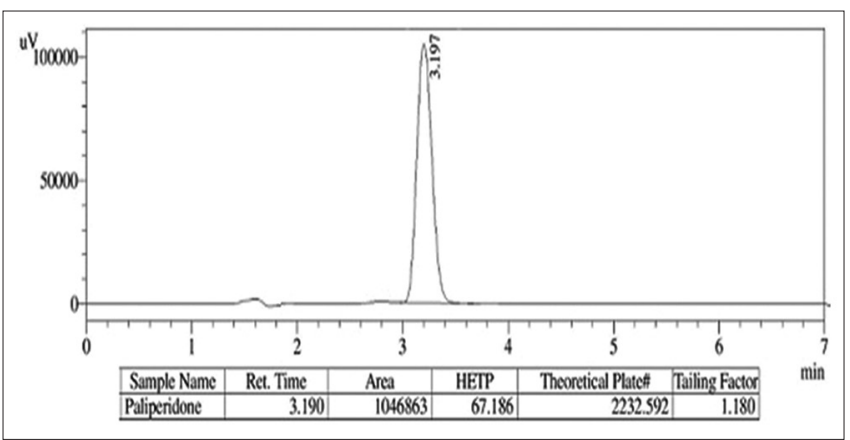

Fig. 3: A typical high-performance liquid chromatography chromatograph of paliperidone

\section{Ex vivo permeation kinetics study}

Ex vivo permeation studies of the paliperidone was performed using modified Franz diffusion cell through rat abdominal skin. The cumulative amount of paliperidone permeated alone and with some permeation enhancers is shown in Fig. 4. The Jss, Kp, ER, and the cumulative amount of drug permeated at $12 \mathrm{~h}$ are summarized in Table 1 . The steady-state flux and permeability coefficient of paliperidone was found to be $3.264 \mu \mathrm{g} / \mathrm{cm}^{2} / \mathrm{h}$ and $1.306 \times 10^{-2} \mathrm{~cm} / \mathrm{h}$, respectively. The enhancement effect of some permeation enhancers on paliperidone through rat abdominal skin was found in the increasing order as follows: Hyaluronidase $>$ DMSO $>$ Groundnut oil $>$ Tween 80 . The ER of pure drug and with enhancers is shown in Fig. 5. The addition of permeation enhancers has increased the rate of skin permeation compared to pure drug. Hyaluronidase has got high permeation enhancing activity than 
other permeation enhancers, with the highest permeation steady-state flux of $12.038 \mu \mathrm{g} / \mathrm{cm}^{2} / \mathrm{h}$ and permeation enhancement rate was 3.69 folds higher than the control (no enhancer) indicating hyaluronidase is to be better permeation enhancer.

\section{Effect of permeation enhancers}

\section{Tween 80}

Tween 80, also known as polysorbate 80 , is a nonionic surfactant and emulsifier used in food and cosmetics industry. Tween 80 is widely used in topical preparations as a solubilizer and skin permeation enhancers. In general, nonionic surfactants possess the least toxicity and have

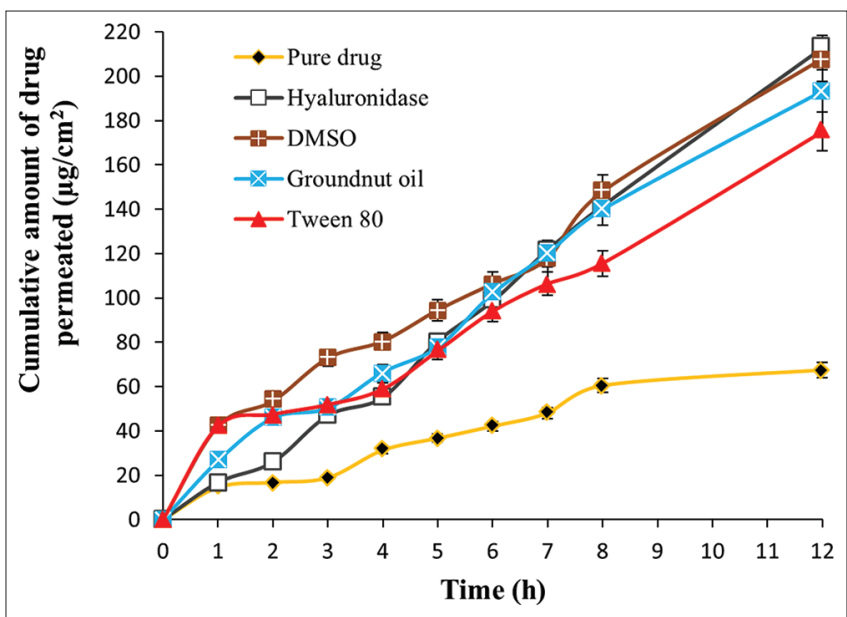

Fig. 4: Cumulative amount of drug permeated, alone and with some permeation enhancers the potential to irritate the skin [26]. The enhancement ability of nonionic surfactant may be through interaction with the stratum corneum by increasing local water concentration with successive swelling and expansion skin thickness is frequently accompanied by the curling of membrane edges [27]. The permeation enhancing activity of the nonionic surfactant tween 80 was attributed may be due to increased fluidity and later solubility and extract of lipid components of stratum corneum by penetration of tween 80 into the intercellular space of stratum corneum. The other mechanism by which permeation enhancing the activity of tween 80 is disruption of the lipid arrangement on the stratum corneum by penetration into the intercellular matrix results in interaction and binding with keratin filaments. It also increases the moisture content of the protein in stratum corneum. This effect is attributed to their amphiphilic nature, where the surfactant hydrophilic groups interact with the keratin domains and their bound water affect aqueous filled spaces that ultimately affect the drug partitioning [28]. In this study, results showed that the paliperidone flux significantly increased by the addition of tween 80 . The ER of tween 80 was 2.59 folds higher than the control; this may be due to a synergistic effect of PEG 400 and tween 80 . The same effect was observed in skin permeation study of diazepam and lorazepam in presence of tween 80 [14,29].

\section{Groundnut oil}

Groundnut oil is a mild-tasting vegetable oil derived from peanuts. The major component of groundnut oil are oleic acid (43\%), linoleic acid (21\%), palmitic acid (10\%), capric acid, lauric acid, stearic acid, arachidic acid, etc. [30] and other fatty acid esters. Oleic acid is the major fatty acid content of the groundnut oil, which increases the skin permeation by acting selectivity on the extracellular lipids by lipid fluidization and lipid phase separation mechanism. Oleic acid appears to decrease the viscosity of lipid at only superficial layers without modifying the conformational order of stratum corneum lipids. Oleic acid also causes lipid phase separation where solid and fluid states

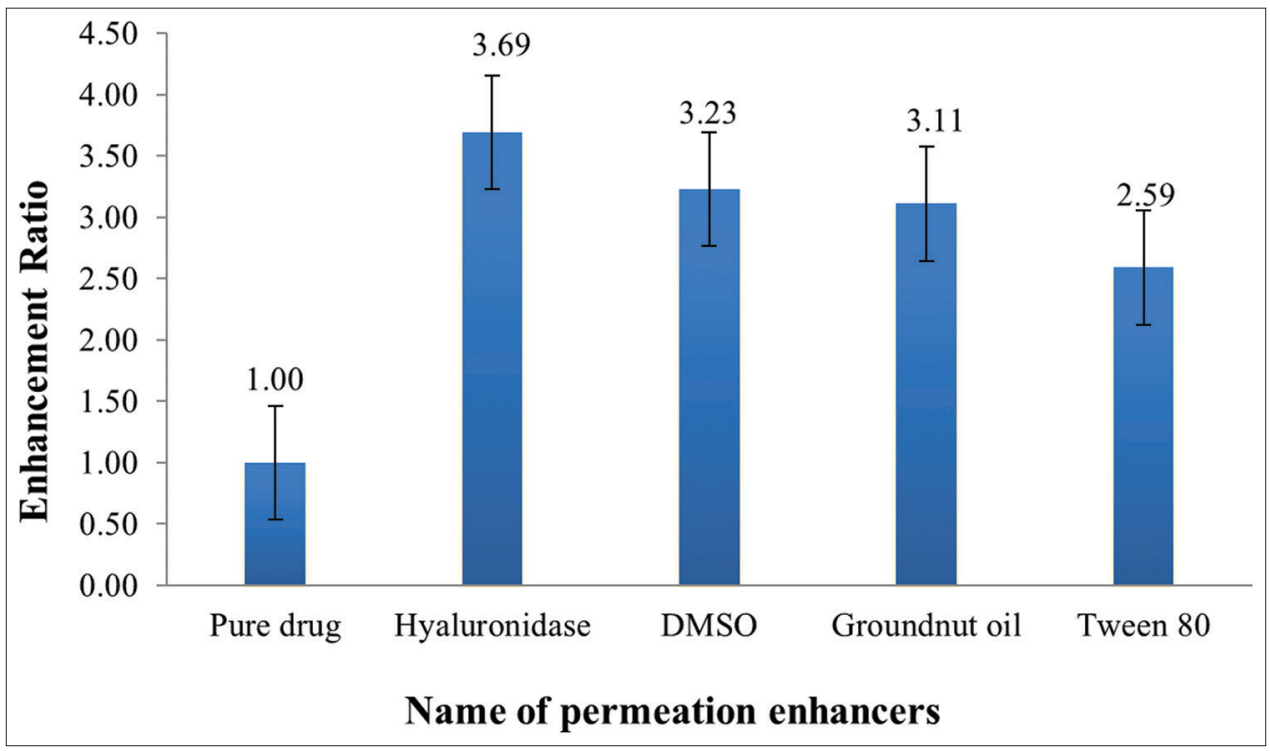

Fig. 5: Enhancement ratio of paliperidone alone and with some permeation enhancers

Table 1: Data for steady-state flux, permeability coefficient, ER, and CADP at $12 \mathrm{~h}$ in the absence and presence of the permeation enhancers (5\%)

\begin{tabular}{|c|c|c|c|c|}
\hline Name of permeation enhancers & Steady-state flux $\left(\mu \mathrm{g} / \mathrm{cm}^{2} / \mathrm{h}\right)$ & $\begin{array}{l}\text { Permeability } \\
\text { coefficient }(\mathrm{cm} / \mathrm{h}) \times 10^{-2}\end{array}$ & $\begin{array}{l}\text { Enhancement } \\
\text { ratio }\end{array}$ & CADP at $12 \mathrm{~h}\left(\mu \mathrm{g} / \mathrm{cm}^{2}\right)$ \\
\hline Pure drug & $3.264 \pm 0318$ & $1.306 \pm 0.127$ & 1.00 & 67.500 \\
\hline Hyaluronidase & $12.038 \pm 0.370$ & $4.815 \pm 0.148$ & 3.69 & 212.760 \\
\hline DMSO & $10.527 \pm 0.717$ & $4.211 \pm 0.285$ & 3.23 & 207.900 \\
\hline Groundnut oil & $10.164 \pm 0.502$ & $4.066 \pm 0.201$ & 3.11 & 193.320 \\
\hline Tween 80 & $8.426 \pm 0.716$ & $2.380 \pm 0.292$ & 2.59 & 174.960 \\
\hline
\end{tabular}

Values are expressed as mean \pm SD $(n=3)$. SD: Standard deviation, CADP: Cumulative amount of drug permeated, DMSO: Dimethyl sulfoxide, ER: Enhancement ratio 
coexist, results in improved skin permeability [31] and their esters like isopropyl myristate [32]. Percutaneous enhancing activity of drugs is more effective in unsaturated fatty acids than saturated fatty acids [33]. The lag time of the drug molecule was reduced in a formulation containing vegetable oils as a permeation enhancing agent, which may be attributed due to a decrease in diffusional path length of the molecule due to changes in stratum corneum $[34,35]$.

\section{DMSO}

DMSO an aprotic polar solvent and is miscible in both polar and non-polar solvents. It is one of the earliest and most widely used chemicals in the pharmaceutical formulation as a penetration enhancer as demonstrating the favorable safety and toxicity profile at low concentration over the past three decades [36]. The ability of DMSO to increase the permeability of both hydrophilic and hydrophobic molecules through the cell membrane is due to change of its position from polar lipid head region to the non-polaric hydrocarbon chain region, and this shift is facilitated by the formation of pores through the skin barrier attributed to enhanced permeation. The other mechanism involved in the enhanced permeation by DMSO is either changing the configuration of the protein structure of the stratum corneum reversibly or by enhancing the thermodynamic activity of the drug [37]. The presence of DMSO in stratum corneum allows the formation of channels due to the swelling in the stratum corneum facilitated decreased diffusional resistance thereby enhancing the permeation [38]. DMSO enhances the drug permeability may be due to the extraction of skin lipids or denaturation of stratum corneum proteins or the formation of hydrogen-bonded complexes with stratum corneum lipids and the distortion [39].

\section{Hyaluronidase}

Among the permeation enhancers tested, an enzyme hyaluronidase impressively enhanced the flux of the paliperidone than other permeation enhancers. It has been approved by USFDA for cosmetic use as an enhancement of SQ absorption [40]. The high spreadability nature of hyaluronidase could be the reason for permeation enhancement of paliperidone. The spreading factor of this enzyme causes local tissue damage, and it is due to the degradation of hyaluronic acid makes a loss of integrity in the extracellular matrix of soft connective tissue leading to enhanced diffusion. The ability of the degraded product of hyaluronic acid to decrease the viscosity of body fluids is also responsible for faster diffusion [41,42]. Hyaluronic acid is a linear polysaccharide and is biosynthesized in situ in humans, and other animals found abundantly in the extracellular matrix due to its biocompatibility, biodegradability, less toxic, and non-irritant [43]. It has been extensively used in TTS as a permeation enhancer

\section{CONCLUSION}

The present research study comprises evaluation of various permeation enhancers which would be used for the preparation of TTS of paliperidone. The received pure drug was identified, and its purity was determined and is at par with the official pharmacopeial limits. The permeation enhancersincluded in the present study were hyaluronidase, DMSO, groundnut oil, and tween 80 and ex vivo skin kinetic permeation experiments were performed with pure drug alone and along with the individual permeation enhancers to obtain steady-state flux which interim gives permeability coefficient, ER and the cumulative amount of drug permeated at $12 \mathrm{~h}$. The results of these parameters suggested that the permeation enhancing activity of permeation enhancers included in the study was in the order: Hyaluronidase $>$ DMSO $>$ Groundnut oil $>$ Tween 80 . Based on this preliminary study results conclude that hyaluronidase would be the suitable permeation enhancing agent for the transdermal therapeutic system of paliperidone.

\section{ACKNOWLEDGMENT}

The authors thank BAL Pharma Ltd., Bengaluru, India, for providing a gift sample of paliperidone.

\section{AUTHOR'S CONTRIBUTIONS}

The first author conducted the experimental studies, data acquisition, data analysis, manuscript preparation, and editing and the second author helped in carrying out experiments, manuscript preparation, and editing. The third author designed and supervised the experimental work, data acquisition, data analysis, preparation of manuscript, and critically reviewed the manuscript for final approval.

\section{CONFLICTS OF INTEREST}

The authors declare that they have no conflicts of interest.

\section{REFERENCES}

1. Chein YW. Novel Drug Delivery Systems Fundamental, Developmental Concepts, Biomedical Assessments. New York: Marcel Dekker Inc.; 1982.

2. Pastore MN, Kalia YN, Horstmann M, Roberts MS. Transdermal patches: History, development and pharmacology. Br J Pharmacol 2015;172:2179-209.

3. Wilson EJ. Three Generations: The Past, Present, and Future of Transdermal Drug Delivery Systems. USA: Pharmcon. SC; 2011.

4. U.S. Food and Drug Administration. Approved Drug Products with Therapeutic Equivalence, Evaluations. Rockville, MD, USA: U.S. Food and Drug Administration; 2016.

5. Singh I, Morris AP. Performance of transdermal therapeutic systems: Effects of biological factors. Int J Pharm Investig 2011;1:4-9.

6. Paudel KS, Milewski M, Swadley CL, Brogden NK, Ghosh P, Stinchcomb AL, et al. Challenges and opportunities in dermal/ transdermal delivery. Ther Deliv 2010;1:109-31.

7. Willams AC, Barry BW. Permeation enhancer. Adv Drug Deliv Rev 2012;64:128-37.

8. Karande P, Mitragotri S. Enhancement of transdermal drug delivery via synergistic action of chemicals. Biochim Biophys Acta 2009;1788:2362-73.

9. Haque T, Talukder MMU. Chemical enhancer: A simplistic way to modulate barrier function of the stratum corneum. Adv Pharm Bull 2018;8:169-79.

10. Ameen D, Michniak-Kohn B. Transdermal delivery of dimethyl fumarate for Alzheimer's disease: Effect of penetration enhancers. Int J Pharm 2017;529:465-73.

11. Sweetman SC, editor. Martindale: The Complete Drug Reference. $34^{\text {th }}$ ed. London: Pharmaceutical Press; 2002.

12. Alphs L, Fu DJ, Turkoz I. Paliperidone for the treatment of schizoaffective disorder. Expert Opin Pharmacother 2016;17:871-83.

13. Peuskens J, Rubio G, Schreiner A. Dosing and switching of paliperidone ER in patients with schizophrenia: Recommendations for clinical practice. Ann Gen Psychiatry 2014;13:10.

14. Prabhakar D, Divya A, Prathyusha R, Kumar K. Augmentation of dissolution profile of poorly soluble paliperidone by employing liquisolid technology. Int J PharmTech Res 2014;6:710-9.

15. Venkatasairam K, Gurupadayya BM, Vishwanathan BI, Chandan RS. Determination of octanol-water partition coefficient of novel coumarin based anticancer compounds by reversed-phase ultra-fast liquid chromatography. Int J Pharm Pharm Sci 2017;9:98-104.

16. Akram MR, Ahmad M, Abrar A, Sarfraz RM, Mahmood A. Formulation design and development of matrix diffusion controlled transdermal drug delivery of glimepiride. Drug Des Devel Ther 2018;12:349-64.

17. Takeuchi H, Mano Y, Terasaka S, Sakurai T, Furuya A, Urano H, et al. Usefulness of rat skin as a substitute for human skin in the in vitro skin permeation study. Exp Anim 2011;60:373-84

18. Davaran S, Rashidi MR, Khandaghi R, Hashemi M. Development of a novel prolonged-release nicotine transdermal patch. Pharmacol Res 2005;51:233-7.

19. Subhash PG, Dinesh BM, Ravikumar M. Feasibility of lercanidipine hydrochloride for TDDS: Permeation kinetic study in presence of various penetration enhancers. Int J Pharm Pharm Sci 2011;3:103-7.

20. Mori NM, Patel P, Sheth NR, Rathod LV, Ashara KC. Fabrication and characterization of film-forming voriconazole transdermal spray for the treatment of fungal infection. Bull Fac Pharm Cairo Univ 2017;55:41-51.

21. Kumar S, Randhawa JK. Solid lipid nanoparticles of stearic acid for the drug delivery of paliperidone. RSC Adv 2015;5:68743-50.

22. Aggarwal G, Dhawan S, Harikumar SL. Formulation, in vitro, and in vivo evaluation of matrix-type transdermal patches containing olanzapine. Pharm Dev Technol 2013;18:916-25.

23. Samanta MK, Dube R, Suresh B. Transdermal drug delivery system of 
haloperidol to overcome self-induced extrapyramidal syndrome. Drug Dev Ind Pharm 2003;29:405-15

24. Chandrashekar NS, Shobha Rani RH. Physicochemical and pharmacokinetic parameters in drug selection and loading for transdermal drug delivery. Indian J Pharm Sci 2008;70:94-6.

25. Rajkumar M, Surendra G. Preparation and characterization of nanocrystals for solubility and dissolution rate enhancement of paliperidone using different hydrophilic carriers: In vitro-in vivo study. Asian J Pharm Clin Res 2018;4:393-8.

26. Pal C, Giri P. Surfactants as penetration enhancer in transdermal drug delivery system. Int $J$ Res Rev 2017;4:104-16.

27. Rhein L, Robbins C, Fernee K. Surfactant structure effects on swelling of isolated human. J Soc Cosmet Chem 1986;37:125-39.

28. Pandey A, Mittal A, Chauhan N, Alam S. Role of surfactants as penetration enhancer in transdermal drug delivery system. J Mol Pharm Org Process Res 2014;2:2-7.

29. Nokhodchi A, Shokri J, Dashbolaghi A, Hassan-Zadeh D, Ghafourian T, Barzegar-Jalali M, et al. The enhancement effect of surfactants on the penetration of lorazepam through rat skin. Int J Pharm 2003;250:359-69.

30. Anyasor GN, Ogunwenmo KO, Oyelana OA, Ajayi D, Dangana J. Chemical analysis of groundnut oil (Arachis hypogaea). Pak J Nutr 2009;3:269-72.

31. Naik A, Pechtold LA, Potts RO, Guy RH. Mechanism of oleic acidinduced skin penetration enhancement in vivo in humans. J Control Release 1995;37:299-306.

32. Fujii M, Hori N, Shiozawa K, Wakabayashi K, Kawahara E, Matsumoto $\mathrm{M}$, et al. Effect of fatty acid esters on permeation of ketoprofen through hairless rat skin. Int J Pharm 2000;205:117-25.

33. Mittal A, Sara UV, Ali A, Aqil M. Status of fatty acids as skin penetration enhancers-a review. Curr Drug Deliv 2009;6:274-9.

34. Aggarwal G, Dhawan S, Hari Kumar SL. Formulation, in vitro and in vivo evaluation of transdermal patches containing risperidone. Drug Dev Ind Pharm 2013;39:39-50.

35. Jain R, Aqil M, Ahad A, Ali A, Khar RK. Basil oil is a promising skin penetration enhancer for transdermal delivery of labetolol hydrochloride. Drug Dev Ind Pharm 2008;34:384-9.

36. Marren K. Dimethyl sulfoxide: An effective penetration enhancer for topical administration of NSAIDs. Phys Sportsmed 2011;39:75-82.

37. Lundborg M, Wennberg CL, Narangifard A, Lindahl E, Norlén L. Predicting drug permeability through skin using molecular dynamics simulation. J Control Release 2018;283:269-79.

38. Zafar S, Ali A, Aqil M, Ahad A. Transdermal drug delivery of labetalol hydrochloride: Feasibility and effect of penetration enhancers. J Pharm Bioallied Sci 2010;2:321-4.

39. Anigbogu AC, Williams AC, Barry BW, Edwards HM. Fourier transform raman spectroscopy of interactions between the penetration enhancer dimethyl sulfoxide and human stratum corneum. Int J Pharm 1997; 155:241-50.

40. Panchagnula R. Transdermal delivery of drugs. Indian J Pharmacol 1997;29:140-56.

41. Girish KS, Kemparaju K. The magic glue hyaluronan and its eraser hyaluronidase: A biological overview. Life Sci 2007;80:1921-43.

42. Kong M, Chen XG, Kweon DK, Park HJ. Investigations on skin permeation of hyaluronic acid based nanoemulsion as transdermal carrier. Carbohyd Polym 2011;2:837-43.

43. Yang R, Wei T, Goldberg H, Wang W, Cullion K, Kohane DS, et al. Getting drugs across biological barriers. Adv Mater 2017;29:1606596. 